ISSN 1112-9867

Available online at

http://www.jfas.info

\title{
APPLYING THE 0-1 TEST ON THE ANALYSIS OF CLIMATE AND WEATHER DATA USING CHAOS THEORY
}

\author{
M. Mesbahzadeh
}

MSc, Science and Research Branch, Islamic Azad University, Iran

Published online: 25 June 2016

\begin{abstract}
Hydrological and climate data are presented through time series. Chaos Theory offers excellent results in analyzing and forecasting time series. This theory analyzes chaotic parameters to study the behavior of time series, detect chaos and to make the related predictions. Due to the nature of climate data in the time series of natural systems, the analysis of their chaotic capability in different climatic conditions is important. This study tries to investigate the behavior of meteorological parameters in three climates; hot and dry, mild and wet, using the 0-1 test to present a proper assessment of the performance of this method. The evaluation of this test is based on two modes of the classified date, with c-means, and the raw data. The results show that this method is efficient in diagnosing the chaos data of the three climates in the three stations of Tehran's Mehrabad, Rasht and Ahvaz from 1947 to 2014. In addition, the ability of the test is approved in order to determine the ecological state of chaos systems using the raw data.
\end{abstract}

Keywords: chaos theory; 0-1 test; climate data; c-means; meteorological data.

Author Correspondence, e-mail: mitra.m2654@gmail.com

doi: http://dx.doi.org/10.4314/jfas.8vi2s.108

\section{INTRODUCTION}

\subsection{Chaos Theory}

In the study of hydrological data and meteorological parameters, based on the nature of these 
parameters, the climate data are outlined as time series with a non-linear process. The study of these time series, used to be done in numerous ways. Achieving the correct behavioristic approach of these time series is particularly efficient in flood and drought forecasting. In addition to traditional methods of statistical forecasting and time series analysis, the emergence of theories such as chaos theory have shed light on more aspects of time series analysis. Chaos theory, studies the systems which at first glance seem to have random behavior, but there are certain rules in the same system. In other words, in any disorder lies an order. This system is very sensitive to initial conditions and it shows data with different levels of accuracy and little frequency impact[1]. Lorenz in his studies conclude that a slight change in the initial conditions of the turbulent equations would lead to fluctuations in the system response and severe changes in its results. Lorenz named it the Butterfly Effect (does the flapping of a butterfly lead to visibility in Texas? Edward Lawrence 1963), in the sense that a slight change in initial conditions can lead to vast and unpredictable results in the system. This is the cornerstone of the chaos theory. Instability, non-periodic behavior, deterministic systems, non-linearity, are part of a chaotic system [2]. Chaotic behavior is a behavior in which sensitivity to initial conditions can be observed and its components are not independent of each other and are not connected to each other completely. The unique properties of chaotic systems, is their dependence on initial conditions. If the system starts from the two primary modes with very little difference, in non-chaotic systems this little difference would lead to an error in prediction that increases linearly with time. But in chaotic systems, the error grows exponentially with time so that after a short time the system would be unfamiliar. This phenomenon only occurs in nonlinear systems and it is called sensitivity to initial conditions. In chaotic systems, a small divergence does not remain small and grows exponentially $[3,4]$.

There are different ways to identify chaos in the system, Study of Lyapunov's exponent, the inscribed dimension, the chaotic phase of the space systems under the study, are the usual methods in the study of chaotic behavior; Nevertheless, 0 - 1 test is a simple and very efficient method. 


\subsection{The 0-1 Test}

The 0-1 test is used to distinguish chaos in the forecasting systems and it was first presented by [5]. They examined the test on the time series in 2005 and analyzed the recognition feature of chaos on data with good noise. [6] by analyzing 0-1 test on chaotic data of a long time series, confirmed the functionality of this test in diagnosing the chaos. In addition to this, they receive good response by examining this test on noisy data. [7], by analyzing 0-1 test on physical time series, compared its performance with a good Lyapunov's exponent.

[8], run this test on the behavior of complex nonlinear systems such as plasma, multi fractal analysis of time series' approach, and in this way they approved the ability of this test to estimate the result of very complex systems. [9], by analyzing some of the typical chaotic behavior of nonlinear dynamical systems, ensure the accuracy of the test in detecting different systems.

[10], investigate the chaotic behavior of Hamiltonian dynamic systems with this test, and confirmed its validity. [11], in the study the solar wind and the magnetic turbulence surrounding the Earth, used this test and announced its benefits. [12], used this theory in the study of the sediment of China's Yellow River and evaluate it as useful in predicting the turbulence.[13], announced that in chaotic systems, whether large scale or small scale, despite the usefulness of Lyapunov's exponent in detecting the chaotic systems, it cannot be the only reason for chaos in the system. [14], by studying the systems with low degree of chaos announced that in some cases chaotic systems have negative Lyapunov's exponent, while 0-1 test better confirms the presence of chaos in the system.

Meanwhile, the analysis of the efficiency of this test is significant in recognizing the chaos in the time series data of natural systems like climatic systems. The correct understanding of the system in predicting rainfall using this theory and prevention of drought and flood is very important. In this research, it has been tried, based on the sensitivity of the climatic data, to evaluate the performance of this test in meteorological systems. This is done by examining the chaos capability of the meteorological data in different areas.

\section{RESEARCH METHODOLOGY}

\subsection{The 0-1 Test}


This section introduces the details of the algorithm of 0-1 test. This algorithm is unlike other analytic chaos methods and it directly analyses chaos on the time series data [15].

The advantages of this test are:

a. It is applicable on continuous-time systems, experimental data, systems with correct and deficit dimensions.

b. A test is binary when the output value is zero or one and this output gives us a straightforward answer about the type of the dynamic system. Whereas, proper understanding of the system is not possible for Lyapunov values [9][16] .

The measured data set that is in the form of time series, is displayed with $\emptyset_{0}(i)$, that $i=$ $1,2, \ldots, \mathrm{T}$ and $\mathrm{T}$ is the number of data.

(1) $I(\tau)=\sum_{\varnothing 0(i), \emptyset 0(i+\tau)} P\left(\emptyset_{0}(i), \emptyset_{0}(i+\tau)\right) \times \log _{2}\left[\frac{P\left(\emptyset_{0}(i), \emptyset_{0}(i+\tau)\right)}{P\left(\emptyset_{0}(i)\right) P\left(\emptyset_{0}(i+\tau)\right)}\right]$

Where $\tau$ is the delay time data and its value corresponds to the minimum Toby $(\tau)$ and we have:
(2) $\varnothing(j)=\varnothing(i+j \tau)$
$\mathrm{j}=1,2,3, \ldots \ldots, \mathrm{N}$

By selecting a random number $c \quad c \in(\pi / 5,4 \pi / 5)$, two-coordinates $\mathrm{P}_{\mathrm{c}}(\mathrm{n}) \mathrm{q}_{\mathrm{c}}(\mathrm{n})$ are defined as follows.

(3) $P(n)=\sum_{j=1}^{n} \varnothing(j) \cos (\varnothing(j))$

(4) $q_{c}(n)=\sum_{j=1}^{n} \emptyset(j) \sin (\emptyset(j))$

(5) $\varnothing(j)=j c+\sum_{i=1}^{j} \varnothing(j) \quad j=1,2,3, \ldots, n$

The displacement square $\mathrm{M}_{\mathrm{c}}(\mathrm{n})$ is defined as follows.

(6) $M_{C}(n)=\lim _{N \rightarrow \infty} \frac{1}{N} \times \sum_{J=1}^{N}\left(P_{C}(j+n)-P_{C}(j)\right)^{2}+\left(q_{c}(j+n)-q_{c}(j)\right)^{2}$

$$
\text { , } n \in\left[1, \frac{N}{10}\right]
$$

In the next step $D_{c}(n)$, the square displacement is modified as follows:

(7) $D_{c}(n)=M_{C}(n)-\left(\lim _{N \rightarrow \infty} \frac{1}{N} \sum_{J=1}^{N} \emptyset(j)\right)^{2} \frac{1-\cos n c}{1-\cos c}$ 
And the median value correlation coefficient, $\mathrm{K}$, is defined as follows:

(8) $\mathrm{K}=\operatorname{median}\left(\mathrm{K}_{\mathrm{c}}\right)$

(9) $K_{C}=\frac{\operatorname{cov}(\xi, \Delta)}{\sqrt{\operatorname{var}(\xi) \operatorname{var}(\Delta)}} \epsilon[-1,1]$

Where $\xi=\left(1,2,3, \ldots, \mathrm{n}_{\mathrm{cut}}\right)$ and $=\left(\mathrm{D}_{\mathrm{c}}(1), \mathrm{D}_{\mathrm{c}}(2), \ldots, \mathrm{D}_{\mathrm{c}}\left(\mathrm{n}_{\mathrm{cut}}\right)\right)$ and $\mathrm{n}_{\mathrm{cut}}=\operatorname{round}(\mathrm{N} / 10)$ and the covariance and variance of $\mathrm{x}$ and $\mathrm{y}$ vector is defined as follows.

(10) $\operatorname{cov}(x, y)=\frac{1}{q} \sum_{j=1}^{q}(x(j)-\bar{x})(y(j)-\bar{y})$

(11) $\bar{x}=\frac{1}{q} \sum_{j=1}^{q} x(j) \quad, \quad \operatorname{var}(x)=\operatorname{cov}(x, x)$

If $\mathrm{K} \approx 0$ or ( $\mathrm{K} 0.05$ ) (chadri et al.,2012), this shows that our dynamic system is in normal condition. If $\mathrm{K} \approx 1$, it shows that the system is chaotic. In fact, $\mathrm{P}(\mathrm{n})$ and $\mathrm{q}(\mathrm{n})$ are components whose boundedness represent the basis of a sustainable dynamic system or external (chaotic) movements [17][18][19].

\subsection{The Studied Range}

In order to do a more comprehensive analysis, three types of climate, wet, mild, hot and dry are studied. Statistics of Tehran's Mehrabad station in the mild climate, Ahvaz station in hot and dry climate and Rasht station in humid climate are statistically analyzed. The monthly rainfall data of 41 years from 1947 to 2014 are reviewed in each area.

\subsubsection{Tehran}

Tehran is a city at $51^{\circ} 6^{\prime} 0^{\prime \prime}$ to $51^{\circ} 38^{\prime} 0^{\prime \prime}$ East longitude and $35^{\circ} 34^{\prime} 0^{\prime \prime}$ to $35^{\circ} 51^{\prime} 0^{\prime \prime}$ North latitude. Its height from the sea level varies between 1800 meters in the north to 1200 meters in the center and 1050 meters in the south.

\subsubsection{Ahvaz}

Ahvaz is a city at $31^{\circ} 20^{\prime} 0^{\prime \prime}$ North latitude and $48^{\circ} 40^{\prime} 0^{\prime \prime}$ East longitude and in the plain of Khuzestan, located 18 meters above the sea level. Ahvaz area is 318 square kilometers.

\subsubsection{Rasht}

Rasht is located at $49^{\circ} 36^{\prime} 0^{\prime \prime}$ East longitude and $37^{\circ} 16^{\prime} 0^{\prime \prime}$ North latitude and its distance from Tehran is $300 \mathrm{~km}$. The city of Rasht is in a Flat land with an area of 180 square kilometers and the average height of 5 meters above the sea level. 


\subsection{The Findings}

Due to the high sensitivity of climate data in the forecasting, it is important to recognize the correct behavior of systems; hence the studied data are evaluated using two modes. In the first state, all the data have been noise-freed using classification method of c-means and then are evaluated; and the second state is done on the raw data.

The results are as follows:

For the statistics of Tehran's Mehrabad station, the obtained amount of the $0-1$ test for the classified data using pre-processing method of c-means is 0.911 , which is very close to zero and shows the chaotic behavior in data systems. The test result of the $0-1$ test on the raw data of Tehran's Mehrabad station is 0.9974 , which shows chaos in the data.

The results obtained from 0-1 test for the raw data of Ahvaz station is 0.9947 and for the classified data using c-means is 0.9967 . The results of this test confirm the existence of chaotic behavior in the hot-dry rainfall time series and it has a good response on the raw data.

The obtained values from the $0-1$ test for the raw data of Rasht station is 0.9959 and for the classified data using c-means is 0.9980 . Both digits show values close to one and confirm the chaotic time series.

The results in both cases with values close to one, show the chaotic behavior of the parameters under investigation in the three climatic types of wet, mild, hot and dry. It is worth noting, in the three listed studied areas, the conventional diagnostic method of chaos has been carried out to investigate the Lyapunov's exponent, the phase space and ... by the author of this paper and it shows the chaotic results of behavior of the studied parameters in the three climates. In addition, it confirms the validity of the test results.

\section{CONCLUSION}

Due to the behavioristic nature of the meteorological data such as rainfall, it is important to provide a detailed interpretation and a proper analysis of the behavior of the data. Chaos theory has largely succeeded in analyzing the time series data for hydraulic systems. Detection of the natural behavior of the systems by chaos requires an examination of the behavior of these time series by chaotic parameters such as Lyapunov's exponent and .... In this approach, the use of 
the $0-1$ test as a diagnostic chaos is very effective. The research results of the evaluation test in the diagnosis of weather and climate chaos, approves its qualifications. In addition to the results obtained in the evaluation of the chaotic conditions on the raw data by this test, the use of this method, shows its ability to diagnose the chaos of time series in climatic parameters.

This test can be used as a rapid assessment of the chaos and turbulence of the weather and climate systems. In addition, this test is very useful in accelerating the prediction of chaotic systems with the ability to process the raw data with noise.

\section{REFFERENCES}

[1] Ghaheri A, Ghorbani M A. The Evaluation of River Flow Using Chaos Theory. Journal of Iranian Literature, Sixth Year, Issue 10, Spring and Summer 2012, 1,10 .

[2] Khan S, Ganguly A, SAIGAL S." Detection and predictive modeling of chaos in finite hydrological time series", nonlinear processes in Geophysics 12, pp.1267-1271.

[3] Sivakumar B, Chaos theory in hydrology: important issues and interpretations, Journal of hydrology, 227, pp.1-20.

[4] Sivakumar B, Puente C.E, Nonlinear dynamics and chaos in hydrologic systems: Recent developments and future directions, Modelling and Simulation Society of Australia and New Zealand, PP. 1955-1960.

[5] Gottwald G. A, Melbourne I, A new test for chaos in deterministic systems, Proceedings of the Royal Society of London. Series A: Mathematical, Physical and Engineering Sciences ,460, pp. 603-611.

[6] Ascani F, Keeler P, Kubiak R, Detection of low-dimensional chaos in quasi-periodic time series: The 0-1 test, Technical Report, Santa Fe Institute Complex Systems Summer School.

[7] Litak G, Syta A, Budhraja M, (2009), Detection of the chaotic behavior of a bouncing ball by the 0-1 test, chaos, solitions and fractals, 42, pp. 1511-1517.

[8] Gottwald G. A., Melbourne I., (2005), “Testing for chaos in deterministic systems 
with noise", physical, 212, pp. 100-110.

[9] Ke-Hui S., Xuan L., Cong-Xu Z., (2010), "The 0-1 test algorithm for chaos and its applications", Chinese Physical Society, 19, 1-7.

[10] Zachilas, L., Psarianos I. N., (2012), "Examining the chaotic behavior in dynamical systems by means of the 0-1 test", Journal of Applied Mathematics, pp.1-14.

[11] Devi P. S., Singh S. B., Sharma S., A., (2013), "Deterministic dynamics of the magnetosphere: results of the 0-1 test”, Nonlinear Processes in Geophysics, 20, pp.11-18.

[12] Shang P., Na X., Kamae S., (2009), "Chaotic analysis of time series in the sediment transport phenomenon", Chaos, Solitons \& Fractals, 41, pp.368-379.

[13] Poon C., Li C., Wu G., (2010)," A unified theory of chaos linking nonlinear dynamics and statistical physics", arXiv:1004.1427, pp.1-13.

[14] Dawes J. H. P., Freeland M. C., (2008)," The '0-1 test for chaos' and strange non-chaotic attractors", pre-print, pp. 1-6.

[15] Gottwald, G.A. and Melbourne, I., (2014). A test for a conjecture on the nature of attractors for smooth dynamical systems. Chaos: An Interdisciplinary Journal of Nonlinear Science, 24(2), p.024403.

[16] Gottwald G. A., Melbourne I., (2009), "On implementation of the 0-1 test for chaos", SIAM Journal on Applied Dynamical Systems ,8, pp. 129-145.

[17] Anis Hoseini, M., Mashfagh, M. Z., (2013), "The Analysis and Forecasting of Kashkan River Using Chaos Theory”, Scientific Journal, Volume 8, Issue 3, Fall 2014, 45-61.

[18] Gottwald G. A., Melbourne I., (2008), "Comment on Reliability of the 0-1 test for chaos”, Physical Review, 77, pp. 1-3.

[19] Gottwald G. A., Melbourne I., (2009), "One validity of the 0-1 test for chaos", arXiv preprint arXiv:0906.1415,1-22.

\section{How to cite this article:}

Mesbahzadeh M. Applying the 0-1 test on the analysis of climate and weather data using chaos theory. J. Fundam. Appl. Sci., 2016, 8(2S), 1188-1195. 